\title{
(2) OPEN ACCESS \\ Dancing Against falls iN Community-dwElling older adults (DANCE): a study protocol of a stratified, block-randomised trial
}

\author{
Mikkel Jacobi Thomsen (1) , ${ }^{1}$ Matthew Liston, ${ }^{2}$ Merete Grothe Christensen, ${ }^{3}$ \\ Peter Vestergaard, ${ }^{3,4,5}$ Rogerio Pessoto Hirata (1) ${ }^{1}$
}

\begin{abstract}
- Additional supplemental material is published online only. To view, please visit the journal online (http://dx.doi. org/10.1136/injuryprev-2021044224).
\end{abstract}

'Department of Health Science and Technology, Aalborg University, Aalborg, Denmark 2Practice and Development, Chartered Society of Physiotherapy, London, UK ${ }^{3}$ Department of Endocrinology, Aalborg University Hospital,

Aalborg, North Denmark Region, Denmark

${ }^{4}$ Department of Clinical Medicine, Aalborg University, Aalborg, Denmark

${ }^{5}$ Steno Diabetes Center North Jutland, Aalborg, Denmark

\section{Correspondence to}

Mikkel Jacobi Thomsen, Department of Health Science and Technology, Aalborg University, Aalborg, Denmark; mjt@hst.aau.dk

Received 17 March 2021 Accepted 30 August 2021

D) Check for updates

(c) Author(s) (or their employer(s)) 2021. Re-use permitted under CC BY-NC. No commercial re-use. See rights and permissions. Published by BMJ.

To cite: Thomsen MJ, Liston M, Christensen MG, et al. Inj Prev Epub ahead of print: [please include Day Month Year]. doi:10.1136/ injuryprev-2021-044224

\begin{abstract}
Background Unintentional falls among older adults are of primary importance due to their impact on quality of life. Falling accounts for $95 \%$ of hip fractures, leading to an times increased risk of death within the first 3 months. Furthermore, physical and cognitive parameters are risk factors for falls. The purpose of this study is to examine the effect of a 6-month salsa dance training intervention, compared with regular fitness circuit training and a control group.

Methods This study will include 180 older adults: 90 healthy patients and 90 patients with osteoporosis. Participants will be allocated randomly in either of the groups, stratified according to age. Training groups will receive 2 weekly 1 -hour training sessions, continuously through 6 months. Participants will be tested at baseline and 6 and 18 months post baseline. Primary outcome will be number of falls and secondary outcomes include bone mineral density, body composition, pain evaluation, weekly physical activity, single-task and dual-task gait patterns, balance, Fullerton Functional Fitness Test and assessment of the mini-BESTest.

Discussion This study will investigate the effects of a specially designed dance training programme (Dancing Against falls iN Community-dwElling older adults (DANCE)) to reduce the risk of falling among older adults. The study will investigate the effect against an active and passive comparator, resulting in the possibility to state, if DANCE training should be an alternative to traditional training.
\end{abstract}

Trial registration number NCT03683849.

\section{BACKGROUND}

Osteoporosis is common in later life, with an estimated $17.7 \%$ of men and $40.8 \%$ of women aged 50 or older being osteoporotic. ${ }^{1}$ Unintentional falls are common among older adults ( $\geq 65$ years), where approximately $30 \%$ experience at least one fall each year. ${ }^{2-5}$ Falls reduce quality of life, ${ }^{6}$ increase the risk of institutionalisation, ${ }^{7}$ result in hospital visits $^{8}$ and in large health expenses. ${ }^{3}$ Approximately $20 \%$ of falls results in serious injuries such as broken bones, head injuries ${ }^{9}{ }^{10}$ or mortality. ${ }^{7}$ Osteoporosis increases the likelihood of fall-related hip fractures three times ${ }^{11}$ compared with those without osteoporosis. It is well established that hip fractures are predominantly caused by falls. ${ }^{12}$ Although only approximately $1 \%$ of all falls result in a hip fracture, ${ }^{13}$ more than $95 \%$ of the hip fractures are caused by falling. The risk of mortality within 3-month post hip fracture is increased, with a pooled relative HR of 5.75 in women and 7.95 in men, ${ }^{14}$ resulting in a 1 -year post-fracture mortality rate of $12 \%-40 \%$ among women. ${ }^{15}$ Seen in the perspective of the incidence rate for osteoporosis in older adults, and the worldwide increase in population of older adults ( $\geq 65$ years) from $8.5 \%$ in 2015 , to $16.7 \%$ in $2050,{ }^{16}$ effective fall prevention programmes are crucial.

The risk of falling has been associated with several intrinsic physical risk factors, such as muscle weakness, ${ }^{17}$ gait deficit, ${ }^{1819}$ balance deficit ${ }^{2021}$ and pain. ${ }^{22}$ When these intrinsic factors are impaired, an extrinsic event, such as slipping or tripping, may result in a fall. ${ }^{23}$ A longitudinal study among community-dwelling older adults found that unintentional/environmental-related falls, such as slipping, sliding and bumping, accounted for 15.8\% of the registered falls, whereas balance/gait impairments, such as tripping, quick movements and lost of balance, accounted for $61.9 \%$ of the falls. ${ }^{24}$ The most frequent activity prior to falling was ambulation, defined as walking, turning and standing, which was performed $56.5 \%$ of the times prior to the fall. Similar results were found in a video capture observational study, showing that slipping and tripping only accounted for $24 \%$ of the registered falls, with the most frequent cause of falling being incorrect weight shifting (41\% of falls), while walking was an activity performed during $48 \%$ of the falls. ${ }^{25}$

Beside physical factors, cognitive impairment has been found to increase the risk of falling. ${ }^{26} 27$ Among patients at a community hospital in the UK, cognitively impaired patients had an OR of $>3$ for falls and injurious falls, ${ }^{27}$ which is similar to results generated from a meta-analysis ${ }^{4}$ (OR of 2.13). In an Intelligence quotient-like scale for cognitive function, based on scores from the Go-No-Go test, Stroop interference test, reaction time and hand-eye coordination errors, an increase of 10 points was associated to a $15 \%$ reduction in fall risk. $^{26}$ A study dividing participants into either having or not having mild cognitive impairments, using the Montreal Cognitive Assessment screening tool found that the participants with an impairment had a larger risk of falling, deduced from a higher Physiological Profile Assessment score and greater postural sway. ${ }^{28}$ Furthermore, Alzheimer's disease has been found to increase the risk of getting a fallrelated hip fracture. ${ }^{29}$ 
Previously, different fall prevention interventions have been examined. ${ }^{30-32}$ The most cost-effective approach $^{32} 33$ has been physical exercise, with the capability of reducing the rate of falling by $23 \% .{ }^{34}$ Physical exercise with a moderate intensity has furthermore been found to reduce hip fracture risk in patients with osteoporosis by more than $40 \% .{ }^{35} 36$ A prospective study found that women with osteoporosis increased their bone mineral density following 12 months of dancing. ${ }^{37}$ Similar results on bone mineral density has been found in a randomised controlled trial (RCT), where the participants performed resistance training, ${ }^{38}$ and in a retrospective cohort study with females performing aerobic dance. ${ }^{39}$ Even though reduced executive function among older adults has been associated with an increased risk of falling, ${ }^{2640}$ only a few focuses on these cognitive factors within fall prevention. ${ }^{41} 42$ A meta-analysis by Hewston and colleagues ${ }^{43}$ concluded that dance probably only have small effects on executive function, but is a promising intervention for improving cognitive health in older adults.

Dancing has previously been shown to improve physical fall-related risk factors among older adults. An RCT on the effect of salsa in healthy older adults showed improvements in gait variables and balance. ${ }^{44}$ Balance was also improved in an RCT on older adults dancing Turkish folklore dance ${ }^{45}$ and lower body function was improved in an RCT study in institutionalised older adults. ${ }^{46}$ Moreover, in an RCT study on older adults, contemporary dancing has been found superior to Tai Chi and strength training in improving switching of attention. ${ }^{47}$ Similarly, in an RCT study with healthy older adults, the dance programme Agilando resulted in enhanced cognitive performance. ${ }^{48}$ In contrast to this, Merom and colleagues ${ }^{49}$ did not find any difference in fall rates between a group performing social dancing and a control group, explaining this by a lack of high balance challenging exercises, such as standing unsupported or on one leg, by including participants with high fall risk at the baseline, and Sherrington and colleagues ${ }^{34}$ stated that effects of programmes primarily consisting of resistance training, dance or walking on the rate of falls are uncommon. In contrast to this, Mattle and colleagues ${ }^{50}$ described in their meta-analysis that dancing reduced rate of falls by $31 \%$ and risk of falling by 37 , although this paper included Tai Chi as a dance-based intervention. Furthermore, Merom and colleagues ${ }^{49}$ stated that there should be an aim to get approximately 56 hours of training in the intervention period, while Granacher et $a l^{44}$ stated that a training period needs to exceed 8 weeks. A review on dance interventions, comparing these to other training genres, does state that dancing is more effective in improving health outcome measures. ${ }^{51}$ However, this review is not made within a specific age group. Furthermore, performing physical activity with others, physical activity encouragement from peers and having a physical activity partner are among the motivational factors for physical exercise in patients with osteoporosis, ${ }^{52}$ and salsa has the possibility to provide all of these three factors.

The municipality of Aalborg is providing strength-related fitness circuit training for older adults within their fall-reduction training services. Previous studies have found lower adherence (56\%-79\%) for strength training ${ }^{53} 54$ than for dancing $(87 \%-$ $92 \%),{ }^{44} 55$ although the 12 -month dance intervention study by Merom and colleagues ${ }^{49}$ did only record $51 \%$ adherence to training. Salsa should be challenging for muscle strength and dynamic balance, as this dance requires frequently changes in direction, ${ }^{44}$ and a similar adaptation in lower body strength between fitness circuit training and dance may occur. The overall reduction in fall rate, due to exercise, has previously been found to be $21 \%-23 \%,{ }^{32} 34$ with the one review stating that balance

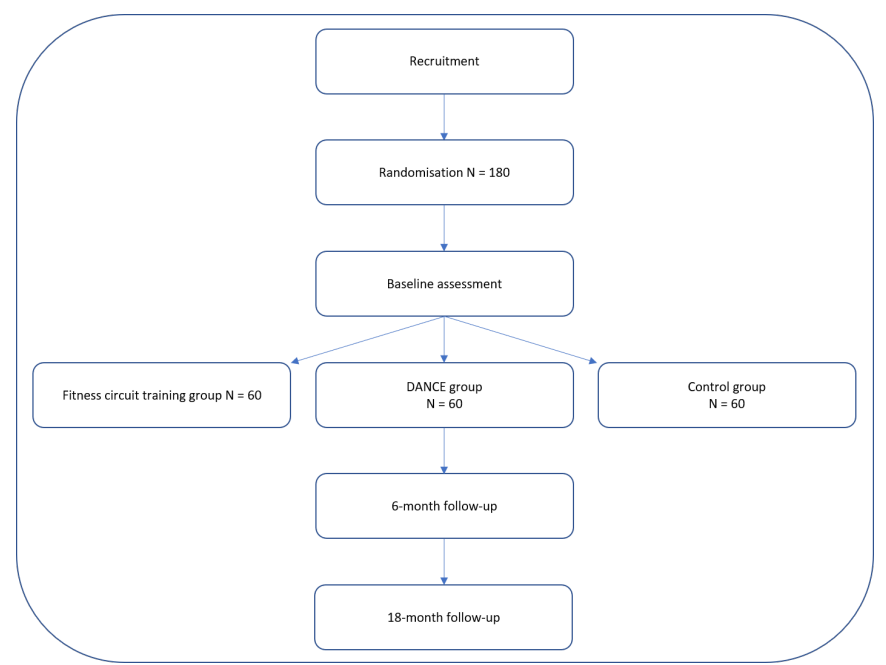

Figure 1 Flowchart of the study flow. DANCE, Dancing Against falls iN Community-dwElling older adults.

challenging exercise forms have larger effects. ${ }^{32}$ The effects of dance training, in comparison to fitness circuit training, has, to the best of our knowledge, not been examined within healthy older adults and older adults with osteoporosis.

The purpose of this study is to examine the effect of a 6-month salsa dance training intervention on the number of falls and fallrelated parameters among participants aged 65 years of age or older. The stratified, block-RCT study will include healthy older adults and older adults with osteoporosis, and compare the salsa training intervention with a passive control group of participants who continues their life as it is, and a group performing fitness circuit training, in order to compare the effect of the dance training to the current training method in the municipality, which have a lower cognitive complexity, and to a group that is not receiving either of them.

We hypothesise that dancing is equally as good, or better, than fitness circuit training at improving the three dominantly fallrelated parameters, ${ }^{56}$ muscle weakness, gait and balance deficits, and that both groups improve at these parameters post training, compared with the control group. Lastly, we hypothesise that the participants in the two training groups will experience fewer fall accidents within the intervention period than the control group.

\section{METHODS}

\section{Study design}

The DANCE study is a stratified, block-randomised, 6-month controlled trial design, which will be conducted to compare three groups: salsa dance training, fitness circuit training and a passive control group (figure 1). Measurements will be taken at baseline, and after 6 and 18 months (see tables 1 and 2). Aalborg University, Aalborg University Hospital and the Municipality of Aalborg will collaborate in this study. Participants will be tested at the Steno Diabetes Center North Jutland at Aalborg University Hospital, Denmark. The protocol is approved by the local ethics committee of Northern Jutland (N-20180065) and registered at clinicaltrials.gov (NCT03683849). The protocol follows the Standard Protocol Items: Recommendations for Interventional Trials (SPIRIT) guidelines ${ }^{57}$ and published results will follow the Consolidated Standards of Reporting Trials (CONSORT) statement. ${ }^{58}$ The administrative information on the DANCE study is given in table 3 . 


\begin{tabular}{|c|c|c|c|}
\hline \multicolumn{4}{|c|}{ Assessments of primary and secondary outcomes } \\
\hline & $\begin{array}{l}\text { Baseline } \\
\text { assessment }\end{array}$ & $\begin{array}{l}\text { 6-month } \\
\text { assessment }\end{array}$ & $\begin{array}{l}\text { 18-month } \\
\text { assessment }\end{array}$ \\
\hline \multicolumn{4}{|l|}{ Demographic data } \\
\hline Height, weight and sex & $\mathrm{x}$ & & $x$ \\
\hline Doctor-prescribed medicine & $x$ & $\mathrm{x}$ & $\mathrm{x}$ \\
\hline Medical history & $\mathrm{x}$ & $\mathrm{x}$ & $\mathrm{x}$ \\
\hline \multicolumn{4}{|l|}{ DXA scan } \\
\hline Bone mineral density & $\mathrm{x}$ & & $\mathrm{X}$ \\
\hline Body composition & $\mathrm{x}$ & & $\mathrm{x}$ \\
\hline \multicolumn{4}{|l|}{ Everyday life assessments } \\
\hline History of falls & $\mathrm{x}$ & $x$ & $x$ \\
\hline Pain evaluation & $x$ & $\mathrm{x}$ & $\mathrm{x}$ \\
\hline Physical Activity Scale for Elderly & $\mathrm{X}$ & $\mathrm{x}$ & $\mathrm{x}$ \\
\hline \multicolumn{4}{|l|}{ Physical and cognitive assessments } \\
\hline $\begin{array}{l}\text { Single-task and dual-task gait } \\
\text { patterns }\end{array}$ & $\mathrm{x}$ & $\mathrm{x}$ & $x$ \\
\hline Static balance on force plate & $\mathrm{x}$ & $\mathrm{X}$ & $\mathrm{x}$ \\
\hline Fullerton Functional Fitness Test & $\mathrm{x}$ & $x$ & $x$ \\
\hline Mini-BESTest & $\mathrm{x}$ & $\mathrm{x}$ & $\mathrm{x}$ \\
\hline
\end{tabular}

DXA, dual-energy $\mathrm{X}$-ray absorptiometry.

\section{Participants}

This study will recruit 180 community-dwelling older adults, 60 for each group. Half of the participants in each group will be healthy older adults, the other half will be patients diagnosed with osteoporosis. Participants will be recruited via advertisements posted at Aalborg University Hospital, nursing homes and activity centres in and around Aalborg, and at Aalborg Senior Sport training sites. Furthermore, recruitment will take place by handing out flyers in public places, at doctors and physiotherapists in and around Aalborg, through information meetings and by advertisement in the press, the radio and at social medias. The wide variety of advertising sites is chosen to target participants or relatives to possible participants.

Participants shall meet the following inclusion criteria:

All participants must be at least 65 years of age, as this is the age at which older adults is defined by the Danish Health Authority $^{59}$ and it makes data comparable to those from the Danish Health Authority ${ }^{60}$ and most of the data included in the
Cochrane libraries review of exercise as fall prevention, ${ }^{34}$ where data from participants 60 years of age or older are included.

Participants will be excluded if they are not capable of performing the six-times 25-metre walking test unaided. Further exclusion criteria are drug addictions, as this may result in varying attendance for training, or uses medical cannabis, as the Danish Medicines Agency has a limited knowledge of side effects of the use of medical cannabis, but states that dizziness is among the known side effects. ${ }^{61}$ Further exclusion criteria are current musculoskeletal injury, current or previous neurologic or mental illnesses, as well as participation in medical trials or other training interventional trials throughout the study period. Lastly, to reduce the risk of fracture-caused dropouts, osteoporosis patients with a T-score lower than -3.5 or patients not receiving medical treatment are excluded, as the risk of getting a vertebral or hip fracture approximately doubles, when T-score is -3.5 compared with $-2.5 .^{62}$ Due to the inclusion and exclusion criteria, none of the participants characterised as being in high risk of falling, when assessed by the Morse Fall scale ${ }^{63}$ or the Hendrich II Fall Risk Model, ${ }^{64}$ were included in the study. On completion of the baseline test, the researchers conducting the test (MSc Sport Science) would assess whether the participant was suitable for receiving dance training.

Potential participants will receive written information about the study by email or regular mail. The written information contained information stating, that there 'is always a risk of falling when conducting these exercise'. Prior to the baseline testing, a member of the research group will provide verbal information, obtain verbal consent and screen for eligibility. Informed written consent will be collected prior to the start of the baseline assessment.

\section{Randomisation}

Participants will be allocated to either of the three groups prior to the baseline assessment, to prevent observer bias. The participants will be divided into two groups, healthy older adults and older adults with osteoporosis. The randomisation within each group will be stratified according to age, to secure an equal mean age. Randomisation will be performed in blocks of nine participant. The nine participants will be divided into blocks of three according to age, youngest, middle and oldest. The ID number of each participant will be placed in a sealed envelope, securing three similar looking envelopes in each age-divided category

Table 2 Schedule for enrolment, allocation, intervention and assessment

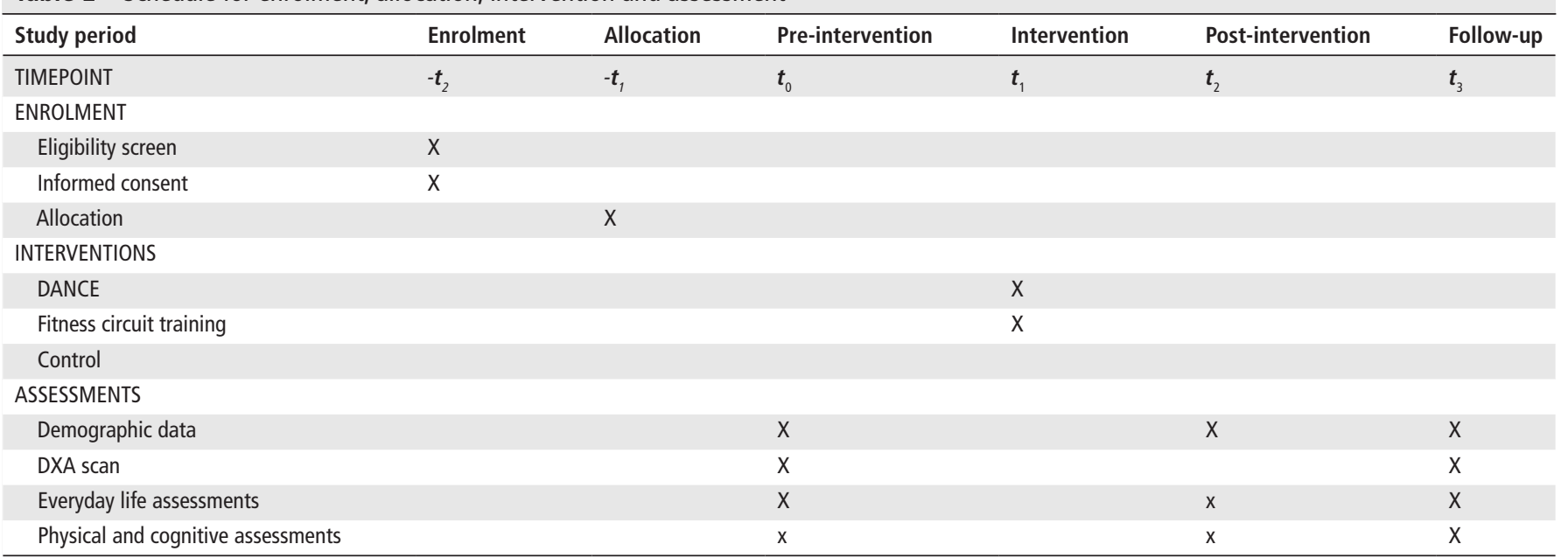

DANCE, Dancing Against falls iN Community-dwElling older adults; DXA, dual-energy X-ray absorptiometry. 
Table 3 Administrative information on the DANCE study

\begin{tabular}{|c|c|c|}
\hline Section/item & Item No & Description \\
\hline Primary registry and trial identifying number & 1 & Clinicaltrials.gov identifier: NCT03683849 \\
\hline Date of registration in primary registry & 2 & 25 September 2018 \\
\hline Secondary identifying numbers & 3 & The North Denmark Region Committee on Health Research Ethics (N-20180065) \\
\hline Source(s) of monetary or material support & 4 & $\begin{array}{l}\text { This study is externally supported by the Trygfonden Foundation (ID: 124404), the Obel family Foundation (No } \\
\text { grant number) and Augustinus fonden (ID 184708) }\end{array}$ \\
\hline Primary sponsor & 5 & Aalborg University, Denmark \\
\hline Secondary sponsor(s) & 6 & $\begin{array}{l}\text { Aalborg University Hospital, Denmark } \\
\text { Aalborg Municipality, Denmark }\end{array}$ \\
\hline Contact for public queries & 7 & $\begin{array}{l}\text { Mikkel Jacobi Thomsen (MJT), PhD student, Cand.Scient. } \\
\text { Phone:+45 } 28127688 \\
\text { Email: mjt@hst.aau.dk }\end{array}$ \\
\hline Contact for scientific queries & 8 & $\begin{array}{l}\text { MJT, PhD student, Cand.Scient. } \\
\text { Phone:+45 } 28127688 \\
\text { Email: mjt@hst.aau.dk }\end{array}$ \\
\hline Public title & 9 & Dancing against fall fractures? \\
\hline Scientific title & 10 & $\begin{array}{l}\text { Dancing Against falls iN Community-dwElling older adults (DANCE): a study protocol of a stratified, block- } \\
\text { randomised trial }\end{array}$ \\
\hline Countries of recruitment & 11 & Denmark \\
\hline Health condition(s) or problem(s) studied & 12 & Falls and fall-related injuries in older adults with and without osteoporosis \\
\hline Intervention(s) & 13 & $\begin{array}{l}\text { Active: } 6 \text {-month salsa dance training } \\
\text { Comparator } 1: 6 \text {-month fitness circuit training } \\
\text { Comparator } 2 \text { : Continuation of regular activity schedule }\end{array}$ \\
\hline Key inclusion and exclusion criteria & 14 & $\begin{array}{l}\text { Ages eligible for the study: } \geq 65 \text { years } \\
\text { Sexes eligible for the study: Both } \\
\text { Accepts healthy volunteers: Yes } \\
\text { Inclusion criteria: (1) Older adults ( } \geq 65 \text { years), (2) osteoporosis with T-score between }-2.5 \text { and }-3.5 \text { OR healthy, } \\
\text { (3) if osteoporosis patient, you must receive medication for osteoporosis } \\
\text { Exclusion criteria: (1) drug addiction, (2) use of medical cannabis, (3) current or previous neurologic, } \\
\text { musculoskeletal or mental illnesses, (4) participation in medical trials or other training interventional trials } \\
\text { throughout the study period, (5) if healthy, use of osteoporosis medication }\end{array}$ \\
\hline Study type & 15 & $\begin{array}{l}\text { Interventional } \\
\text { Allocation: Blocked, stratified randomisation } \\
\text { Intervention model: Parallel assignment } \\
\text { Primary purpose: Prevention }\end{array}$ \\
\hline Date of first enrolment & 16 & January 2019 \\
\hline Target sample size & 17 & 180 \\
\hline Recruitment status & 18 & Recruiting \\
\hline Primary outcome(s) & 19 & $\begin{array}{l}\text { Outcome: Fall rate } \\
\text { Metric/method of measurements: Questionnaire } \\
\text { Timepoint: } 18 \text { months following the baseline assessments }\end{array}$ \\
\hline Key secondary outcomes & 20 & Differences between objective measures and self-reported level of physical activity \\
\hline
\end{tabular}

(youngest, middle and oldest). Within each category, the envelopes will be shuffled and afterwards drawn one by one. The participant which ID number is in the first envelope drawn in each of the age categories will be allocated to the control group, the second to the strength training group and the last to the dance training group. The procedure will be performed by a person within the research group.

\section{Procedure}

An overall structure of the study can be found in figure 1. Participants will be assessed in regard to inclusion and exclusion criteria via phone or email. After being allocated in one of the three groups, participants will undergo baseline testing, where gender, age, height and weight will be recorded, as well as testing of several physical parameters. Participants in the two training groups will be assigned to 1-hour training session twice a week, for 6 months. Follow-up testing will be performed at 6 months (on completion of training) and at 18 months. The assessor of the test will be a person within the research group. This person is not blinded to the randomisation. Participants will be contacted by phone or email to arrange tests at 6 and 18 months.

\section{Intervention}

Every participant is encouraged to continue their everyday life and routine, including self-administered physical exercise, and the training in the two training groups will therefore be seen as add-on to their normal physical exercise, and not a substitute. Attendance to training will be recorded after each training by the dance/fitness instructor. All training will be performed in groups of maximal 20 persons and supervised by an experienced dance teacher or a person with education within fitness exercise, as a previous study has found, that adherence to training is larger when class cohesion is high. ${ }^{65}$ Furthermore, the dance teacher should have prior experience in training older adults and have first aid skills. Prior to the start of the training, the dance instructor will attend meetings with the purpose of providing the instructor with knowledge regarding risk factors associated with osteoporosis. 


\section{Fitness circuit training}

Training following the NEMEX-TJR protocol ${ }^{66}$ is part of the health services at Aalborg municipality. A modified version of this has been used as the fitness circuit training, to mimic the training performed in the municipality. The exercises in circle 4 of the NEMEX-TJR protocol has been modified, as the chair stand is changed to body-weighted squats, and the stair climbing is changed to standing heel raises. The chair stand is a part of the test protocol, and to ensure that the fitness circuit training group does not perform weekly training of a test from the test protocol, this exercise is changed. Stair climbing is changed due to limitations in equipment.

\section{DANCE training}

The aim of developing the DANCE training protocol is to develop a progressive salsa training intervention, focusing on the mechanisms responsible for falling, found by Robinovitch et al. ${ }^{25}$ These are forward, backwards and sideways walking, as well as shift of bodyweight. Furthermore, the steps should be training the most common risk factors for falling, such as muscle strength, gait and balance. ${ }^{56}$

In order to develop the DANCE programme, the researchers (MSc Sport Science) and two skilled salsa dancers divided different salsa dance steps into the aforementioned categories, forward, backwards and sideways walking, as well as shift of bodyweight through twists and turns, and ranked the steps within each category in regard to complexity, difficulty and their ability to challenge the postural control by decreasing the base of support, and therefore move the limit of stability closer to the centre of mass, in order to secure, that each category is trained within each training stage. Each stage should also include steps that mimics walking, as salsa steps with forward walking resembles the initiation and approximately a quarter of the range of motion of lunges, which may decrease the age-related muscle weaknesses in the lower extremities.

The salsa training is divided into four progressive stages. Participants shall master the steps in a stage, before going to the next stage. Two training groups may therefore not be at the same stage, after a specific number of training sessions, as the DANCE training teacher have to evaluate prior to each training, if the group is ready to continue to the next stage. Stage 2 will serve as an add-on to stage 1 , stage 3 an add-on to stage 1 and 2 . Stage 4 is a stage where all the steps in the previous stages are used in a so-called Rueda de casino. The steps are first trained individually and afterwards in pairs. Furthermore, each dance session is started by a warm up, containing known dance steps, without a partner, in order to accommodate shortcomings in the study by Merom et $a l,{ }^{49}$ stating that the balance was not challenged enough in their study, as the dance did not provide participants with unsupported standing. As the person who dance the follower role is provided with larger balance challenges, through more twists and turns, participants will be instructed into changing between dancing leader and follower roles, and to switch partner multiple times during a DANCE training session. On completion of the training, the instructor would record which of the four DANCE training stages the training had been carried out at. To ensure that the training followed the designed programme, at least one training every month would be supervised by a member of the research group.

The four stages contain the following salsa steps:

- Stage 1: Basic step, back basic, sidestep, cuban open step.

- Stage 2: Right turn, dile que no, cradle.

- Stage 3: Enchufla, triple turn, sombrero.
Stage 4: Rueda de casino.

Basic step: Step forward with left foot, back to base, backward with right foot and return to base. Performed in a straight line.

Back basic: Step backward with left foot, back to base, backward with right foot and return to base. Performed in a straight line.

Sidestep: Step sideways with left foot, back to base, sideways with right foot and return to base. Performed in a straight line.

Cuban open style: Step back with left foot while turning the upper body 90 degrees (right foot stays in place), back to base, step back with right foot while turning the upper body 90 degrees (left foot stays in place) and return to base.

Right turn: Leader dances basic steps, follower steps forward on left, turns clockwise and returns to base.

Dile que no: In this step, the leader and the follower will switch place during the step. The leaders steps divide the turn into two 90 degree turns, while the follower performs a 180 degree turn in the end.

Cradle: In this step, the leader dances back basic steps. The follower makes two 180 degree turns. The first turn is counterclockwise and turns the follower with the back toward the leader, before turning clockwise back into the base.

Enchufla: Leader and follower will switch place in this step. Leader will take a little step sideway, going forward on right and turn 180 degrees clockwise, before dancing the last part of a back basic. While the leader performs a clockwise turn, the follower does a counterclockwise turn.

Triple turn: This step starts with the entire step 'Right turn'. Hereafter, the leader makes a turn similar to the one that the follower just did, followed by a third turn, performed by the follower.

Sombrero: Here, the leaders perform the footwork of an entire basic step, while the follower performs a 360 degree clockwise turn. Hereafter, they will perform a 'dile que no'. While performing this step, the arms will be looping across the partners upper body.

Rueda de casino: Here, the dancers are positioned in a circle, performing known steps, called out by the instructor. In this dance, the follower and leaders switches partners frequently.

Participants are taught traditional holds in the dance, but are advised to deviate from these, if they are feeling uncomfortable while performing these.

Within each stage, the difficulty can be manipulated, by changing the music to a song with a higher or lower amount of beats per minute.

Due to the relatively few different steps in each stage, the DANCE training programme is a ready-to-start training protocol, accessible to the municipality of Aalborg and other interested parties. Sound equipment is the only equipment needed to start the intervention. It was not possible to test prior to the intervention, whether the stages were capable of providing the older adult participants with a sufficient degree of difficulty.

\section{Harms}

The risks of adverse reactions from the dual-energy X-ray absorptiometry (DXA) scanning is minimal due to the low dose of radiation applied through the test. X-ray dose is $<100$ microSv per scan, which by two scans is $<200$ microSv or $<0.2 \mathrm{mSv}$. In theory, this will increase the lifetime risk of cancer from approximately 25\% to less than $25.00002 \%$.

Participants are encouraged to report any adverse effects occurring during the entire enrolment in the study. Serious 
adverse effects will be reported to the local ethics committee, and re-evaluation of the protocol will be made.

\section{Data management}

All participants will receive an individual ID number. Data storage will be made using this ID number. All handwritten notes and assessments, as well as the signed, written consent, will be stored in a double locked area, in an area of limited access. The Danish Act on Processing of Personal Data will be complied with, and the collection and storage of data are approved by the Danish Data Protection Agency.

\section{Outcomes}

All tests will be assessed at Steno Diabetes Center North Jutland, Aalborg University Hospital. The participants will go through a 3 -hour session at the baseline, 6-month and 18 -month test. The DXA scan will not be performed at 6 months, in order to minimise the adverse effects of radiation. During baseline assessment, height, weight, sex, intake of doctor-prescribed medicine and medical history will be recorded. Table 1 provides an overview of all the tests and timepoints for assessment. All DXA scans will be examined by a Chief Physician from Steno Diabetes Center North Jutland, Aalborg University Hospital.

\section{Primary outcome measure}

Number of falls is the primary outcome. Falls will be assessed at each of the three tests. A fall is, in Lamb et $a l^{67}$ (p1619), defined as 'an unexpected event in which the participants come to rest on the ground, floor, or lower level'.

\section{Secondary outcome measures}

A wide variety of secondary outcomes will be assessed in the present study, these being physical and cognitive measurement, all previously related to the risk of falling. In addition, a DXA scan will be performed to determine the bone mineral density and body composition of the participants.

DXA scan: Participants will undergo a bone mineral scan for lumbar spine (L1-L4), femoral neck and total hip, performed using a DXA scan. The reported results from the scan are bone mineral density $\left(\mathrm{g} / \mathrm{cm}^{2}\right)$ and T-score. The DXA scan will furthermore perform a body composition scan, with the fat mass (g), fat percentage, lean + bone mineral content $(\mathrm{g})$ and lean +bone mineral content percentage as results for further analysis. A daily quality control programme was in place and coefficient of variation $(\mathrm{CV})$ was: lumbar spine: $\mathrm{CV} \%=1.091 \%$; total hip: $\mathrm{CV} \%=1.15 \%$; femoral neck: $\mathrm{CV} \%=1.77 \% .{ }^{68}$

Pain perception: Participants will be asked to rate the level of pain they feel during a normal day, using a $100 \mathrm{~mm}$ Visual Analogue Scale, with terminal descriptors being 'no pain' and 'worst pain possible'. If the rating is $>0 \mathrm{~mm}$, participants are asked to draw the pain-affected areas on a body chart, and fill in the Danish version of the McGill Pain Questionnaire. ${ }^{69}$

Physical activity level: The general level of physical activity will be assessed by filling out the Physical Activity Scale for Elderly ${ }^{70}$ which is a brief and easily scored survey, shown to be a valid way to measure physical activity in older adults. ${ }^{71}$

Gait stability: The gait pattern of the participants will be assessed using the full-body motion tracking Xsens Awinda (Awinda, Xsens Technologies B.V., Enschede, The Netherlands) solution. Participants will be asked to walk in their normal pace on a 25 -metre path six times. Three of the six times will be a normal-walking single-task test, whereas the remaining three times will be a dual-task test, including counting backwards in steps of 7 , starting from a random number between 150 and 500 , as this dual-task gait test has been found to provide insight into the mechanism involved in the motor control of locomotion. ${ }^{72}$ The variables being recorded for further analysis are gait speed $(\mathrm{m} / \mathrm{s})$, length of a gait cycle $(\mathrm{m})$, gait cycle time $(\mathrm{ms})$ and centre of mass displacement during walking. Furthermore, quantification of the dual-task test will include the total number of subtractions, total number and percentage of successful subtractions, in order to access cognition.

Balance stability: Static balance will be assessed using a force platform. Participants will be asked to stand on a force platform, as quiet as possible, during twelve 30 -second tests. The twelve tests include three tests at each of four conditions, these being:

(1) eyes open on a firm surface, (2) eyes closed on a firm surface, (3) eyes open on a soft surface and (4) eyes closed on a soft surface. The area and velocity of the centre of pressure will be being used for further analysis. ${ }^{73}$

Clinical tests: Participants will be assess using two test batteries capable of being performed in clinical setting, the Fullerton Functional Fitness test ${ }^{74}$ and the mini-BESTest, ${ }^{75}$ as the latter one has been found an accurate tool for identifying older adults with a history of falling. ${ }^{76}$ The Functional Fitness test consist of six tests, testing lower and upper body strength and flexibility, physical stamina and speed, agility and balance during walking. Each test gives an individual score, which is not being calculated to a single score for the entire test. The mini-BESTest consists of 14 tests, testing anticipatory and reactive postural control, sensory orientation and dynamic gait. Each of the 14 tests are rate between 0 and 2, giving a total score between 0 and 28 .

\section{Sample size}

For our sample size calculations, results from Merom and colleagues $^{49}$ were used. Here, when consulting the results from the online supplemental table S2, we see that ballroom dancers with high attendance had a fall rate per person-year of 0.66 , whereas the control group had a fall rate of 0.8 , resulting in a reduction in falls per person-years by $17.5 \%$. We will use a more conservative reduction, $10 \%$, as our intervention has 52 training sessions, whereas the study by Merom and colleagues had 80 . A total sample size of 120 participants would be enough to identify at least $10 \%$ reduction in falls per person-year $(80 \%$ power, alpha of 5\%,20\% dropout rate) when comparing two groups. This study will recruit 120 participants for the two intervention groups, divided into two equally size groups, dance training and fitness circuit training. Furthermore, 60 participants will be recruited for a control group.

\section{Statistical analysis}

The demographic data (gender, age, height, weight) will be assessed with independent samples t-test in regard to fall accidents, in order to test if these data influenced the possible difference between groups. The primary outcome measure, unintentional falls, will be assessed using intention to treat analysis and a negative binomial regression model. Furthermore, the risk ratio and 95\% CIs will be calculated. Within the variables Falls and Osteoporosis, a contingency table with a Pearson's $\chi^{2}$ test will be made.

The secondary outcomes will be tested for normality using a Shapiro-Wilks test and visually investigated using QQ-plots and histograms. A Maunchly's sphericity test will be performed. If sphericity is not violated, a mixed model analysis of variance will be administered, with a Bonferroni-corrected post hoc test. 
In case of violation of sphericity, the Greenhouse-Geisser corrections will be made on the df.

\section{DISCUSSION}

Falls are common among older adults and have high associated healthcare costs (more than $\$ 50$ billion in the USA in 2015). ${ }^{3}$ With an expected doubling in population of older adults $(\geq 65$ years) worldwide from 2015 to $2050,{ }^{16}$ effective and easy implementable fall-preventing interventions are of highest importance. The DANCE intervention is a low-cost alternative to current fall prevention programmes that combines physical and cognitive aspects. The authors therefore believe that it may serve as an effective alternative to traditional training, which lack of focus on cognition as a risk factor, may be the reason for suboptimal improvements. ${ }^{34}$ Since the factors 'social benefits' and 'enjoyment' have both been found to be motivational factors for physical activity among older adults, ${ }^{77}$ with music facilitating increasing exercise participation and adherence, ${ }^{78}$ the DANCE intervention further has the ability to serve as an engaging training tool. As stated in the recommendations for fall prevention, ${ }^{32}$ ongoing participation in exercise is necessary, and we believe that the DANCE intervention, due to the aforementioned motivational factors, can be an ongoing exercise among the participants on completion of the study.

The objective of this stratified, block-randomised trial is to evaluate the effect of the DANCE intervention on number of falls and parameters associated with the risk of falling in adults 65 years of age or older. The study involves both an active and a passive comparator, with the active comparator being fitness circuit training comparable to the training provided by the Municipality in Aalborg. The study therefore has the ability to access the effect in relation to no additional exercise and in relation to traditional training.

\section{PUBLIC INVOLVEMENT}

Prior to the start of the RCT, three pilot studies were conducted. In all three, Aalborg SeniorSport, an organisation providing a

\section{Strengths and limitations of this study}

- If the DANCE (Dancing Against falls iN Community-dwElling older adults) training group is lowering their fall rate or risk of falling, a ready-to-start training protocol is accessible to the municipality of Aalborg and other interested parties.

- The DANCE training protocol is based on the latest recommendations for fall prevention.

- The study further examines if the training currently offered in the municipality has a preventive effect.

- The randomised controlled trial consists of both an active and a passive comparator, making it possible to conclude on the effect in relation to normal prescribed training and no extra exercise.

- The 6-month long training intervention, combined with voluntary participation, may result in a non-direct comparative study participants sample, compared with the population in Denmark, as only relative fit older adults may sign up.

- Fall data are only collected at testing days. Other, superior, methods for collecting fall data exist.

- Due to limited resource, a single member of the research group, who conducted some of the tests, was not blinded to the randomisation. broad variety of sports and physical exercise offers for older adults in Aalborg, were involved in recruiting participants. Through these pilot studies, both the salsa dancing and the test methods were tested and evaluated. The dance was quoted to provide 'joy through the movements', and the sporting director stated that 'all of the participants wanted to continue dancing'. Furthermore, the Municipality of Aalborg is involved in the study by providing training facilities at their activity centres, securing visibility of the study for the older citizens in Aalborg.

\section{DISSEMINATION}

Participants are expected to complete the follow-up at 18 months by December 2022. Publications on the full results will be ready for submission in the spring of 2023. A relevant peer-reviewed scientific journal will be chosen for publication of result from the present study. Press releases will be provided to local and national newspapers, radio and television stations in layman's term to address the general public. Furthermore, workshops and seminars for the general public will be held.

Acknowledgements The authors greatly thank the involved dance instructor, Ms Birgitte Bisgaard Nielsen. A thank you to Ms Mette Brodersen, Ms Line Rosengreen Kaldahl and Ms Ingelise Leegaard, all from Department of Endocrinology, Aalborg University Hospital, for their expertise and help performing DXA scans. A great thanks to Dr Morten Villumsen, Department of Elderly and Health, Section of Training and Activity, Aalborg Municipality, for his help in the collaboration with the activity centres in the municipality. Finally, a great thanks to the employees at Den Rytmiske I Nordkraft and the activity centers in Aalborg Municipality for their assistance during recruitment and for being helpful with training facilities.

Contributors The following has participated in the study design (MJT, RPH, PV, MGC and ML), data acquisition (MJT, RPH and MGC), conduction of training interventions (MJT, RPH and external instructor), analysis of data (MJT and RPH), interpretation of data (MJT, RPH, PV, MGC and ML), draft of the manuscript (MJT, ML, MGC PV, RPH). All authors will read, comment and approve the final manuscript. Authorship are determined following the Vancouver rules.

Funding The study received funding from Department of Health Science and Technology at Aalborg University, TrygFonden (Grant number: 124404), Augustinus fonden (Grant number: 18-4708) and Det Obelske familie Fond (no grant number). The funding will be used to pay the dance instructor, salaries for the investigators, salary for participants in the control group, materials and other overhead expenses. The Municipality of Aalborg and Den Rytmiske I Nordkraft provide training facilities for the training groups. None of the sponsors have financial conflicts of interest in the study. During the study period, continuously fund applications will be made.

Competing interests None declared.

Patient consent for publication Not required.

Ethics approval This study is approved by the local Ethics Committee (N20180065) and conforms to the principles of the Declaration of Helsinki.

Provenance and peer review Not commissioned; externally peer reviewed.

Data availability statement Data sharing not applicable as no datasets generated and/or analysed for this study.

Supplemental material This content has been supplied by the author(s). It has not been vetted by BMJ Publishing Group Limited (BMJ) and may not have been peer-reviewed. Any opinions or recommendations discussed are solely those of the author(s) and are not endorsed by BMJ. BMJ disclaims all liability and responsibility arising from any reliance placed on the content. Where the content includes any translated material, BMJ does not warrant the accuracy and reliability of the translations (including but not limited to local regulations, clinical guidelines, terminology, drug names and drug dosages), and is not responsible for any error and/or omissions arising from translation and adaptation or otherwise.

Open access This is an open access article distributed in accordance with the Creative Commons Attribution Non Commercial (CC BY-NC 4.0) license, which permits others to distribute, remix, adapt, build upon this work non-commercially, and license their derivative works on different terms, provided the original work is properly cited, appropriate credit is given, any changes made indicated, and the use is non-commercial. See: http://creativecommons.org/licenses/by-nc/4.0/.

\section{ORCID iDs}

Mikkel Jacobi Thomsen http://orcid.org/0000-0003-3880-9932 
prevent falls? A systematic review of economic evaluations of falls prevention interventions. Br J Sports Med 2010;44:80-9.

34 Sherrington C, Fairhall NJ, Wallbank GK, et al. Exercise for preventing falls in older people living in the community. Cochrane Database Syst Rev 2019;1:CD012424.

\section{REFERENCES}

1 Vestergaard P, Rejnmark L, Mosekilde L. Osteoporosis is markedly underdiagnosed: a nationwide study from Denmark. Osteoporos Int 2005;16:134-41.

2 Bergen G, Stevens MR, Burns ER. Falls and Fall Injuries Among Adults Aged $\geq 65$ Years - United States, 2014. MMWR Morb Mortal Wkly Rep 2016;65:993-8.

3 Florence CS, Bergen G, Atherly A, et al. Medical costs of fatal and nonfatal falls in older adults. J Am Geriatr Soc 2018;66:693-8.

4 Muir SW, Gopaul K, Montero Odasso MM. The role of cognitive impairment in fall risk among older adults: a systematic review and meta-analysis. Age Ageing 2012:41:299-308.

5 Tinetti ME, Speechley M, Ginter SF. Risk factors for falls among elderly persons living in the community. N Eng/ J Med 1988:319:1701-7.

6 Fuller GF. Falls in the elderly. Am Fam Physician 2000;61:2159-68.

7 Tinetti ME, Williams CS. Falls, injuries due to falls, and the risk of admission to a nursing home. N Engl J Med 1997;337:1279-84.

8 Høidrup S, Sørensen TIA, Grønbaek M, et al. Incidence and characteristics of falls leading to hospital treatment: a one-year population surveillance study of the Danish population aged 45 years and over. Scand J Public Health 2003;31:24-30.

9 Sterling DA, O'Connor JA, Bonadies J. Geriatric falls: injury severity is high and disproportionate to mechanism. J Trauma 2001;50:116-9.

10 Alexander BH, Rivara FP, Wolf ME. The cost and frequency of hospitalization for fallrelated injuries in older adults. Am J Public Health 1992;82:1020-3.

11 Wainwright SA, Marshall LM, Ensrud KE, et al. Hip fracture in women without osteoporosis. J Clin Endocrinol Metab 2005;90:2787-93.

12 Hayes WC, Myers ER, Morris JN, et al. Impact near the hip dominates fracture risk in elderly nursing home residents who fall. Calcif Tissue Int 1993;52:192-8.

13 Cummings SR, Melton LJ. Epidemiology and outcomes of osteoporotic fractures. Lancet 2002;359:1761-7.

14 Abrahamsen B, van Staa T, Ariely R, et al. Excess mortality following hip fracture: a systematic epidemiological review. Osteoporos Int 2009;20:1633-50.

15 Gregg EW, Pereira MA, Caspersen CJ. Physical activity, falls, and fractures among older adults: a review of the epidemiologic evidence. J Am Geriatr Soc 2000;48:883-93.

16 He W, Goodkind D, Kowal P. An aging world 2015, International population reports United States census bureau - March 2016. Aging 2016:165.

17 Moreland JD, Richardson JA, Goldsmith CH, et al. Muscle weakness and falls in older adults: a systematic review and meta-analysis. J Am Geriatr Soc 2004;52:1121-9.

18 Hausdorff JM, Rios DA, Edelberg HK. Gait variability and fall risk in community-living older adults: a 1-year prospective study. Arch Phys Med Rehabil 2001;82:1050-6.

19 Hausdorff JM, Edelberg HK, Mitchell SL, et al. Increased gait unsteadiness in community-dwelling elderly fallers. Arch Phys Med Rehabil 1997;78:278-83.

20 Pfeifer M, Begerow B, Minne HW, et al. Vitamin D status, trunk muscle strength, body sway, falls, and fractures among 237 postmenopausal women with osteoporosis. Exp Clin Endocrinol Diabetes 2001;109:87-92.

21 Topper AK, Maki BE, Holliday PJ. Are activity-based assessments of balance and gait in the elderly predictive of risk of falling and/or type of fall? J Am Geriatr Soc 1993;41:479-87.

22 Leveille SG, Bean J, Bandeen-Roche K, et al. Musculoskeletal pain and risk for falls in older disabled women living in the community. J Am Geriatr Soc 2002;50:671-8.

23 Dionyssiotis Y. Analyzing the problem of falls among older people. Int J Gen Med 2012;5:805-13.

24 Talbot LA, Musiol RJ, Witham EK, et al. Falls in young, middle-aged and older community dwelling adults: perceived cause, environmental factors and injury. BMC Public Health 2005:5:86.

25 Robinovitch SN, Feldman F, Yang Y, et al. Video capture of the circumstances of falls in elderly people residing in long-term care: an observational study. Lancet 2013;381:47-54.

26 Mirelman A, Herman T, Brozgol M, et al. Executive function and falls in older adults: new findings from a five-year prospective study link fall risk to cognition. PLoS One 2012;7:e40297.

27 Vassallo M, Mallela SK, Williams A, et al. Fall risk factors in elderly patients with cognitive impairment on rehabilitation wards. Geriatr Gerontol Int 2009;9:41-6.

28 Liu-Ambrose TY, Ashe MC, Graf $\mathrm{P}$, et al. Increased risk of falling in older communitydwelling women with mild cognitive impairment. Phys Ther 2008;88:1482-91.

29 Weller I, Schatzker J. Hip fractures and Alzheimer's disease in elderly institutionalized Canadians. Ann Epidemio/ 2004;14:319-24.

30 Rubenstein LZ. Falls in older people: epidemiology, risk factors and strategies for prevention. Age Ageing 2006;35:ii37-41.

31 Gillespie LD, Robertson MC, Gillespie WJ, et al. Interventions for preventing falls in older people living in the community. Cochrane Database Syst Rev 2012;2012:CD007146

32 Sherrington C, Michaleff ZA, Fairhall N, et al. Exercise to prevent falls in older adults: an updated systematic review and meta-analysis. Br J Sports Med 2017:51:1750-8.

33 Davis JC, Robertson MC, Ashe MC, et al. Does a home-based strength and balance programme in people aged $>$ or $=80$ years provide the best value for money to
35 Gregg EW. Physical activity and osteoporotic fracture risk in older women. Ann Intern Med 1998;129:81.

36 Moayyeri A. The association between physical activity and osteoporotic fractures: a review of the evidence and implications for future research. Ann Epidemiol 2008; 18:827-35

37 Kudlacek S, Pietschmann F, Bernecker P, et al. The impact of a senior dancing program on spinal and peripheral bone mass. Am J Phys Med Rehabil 1997;76:477-81.

38 Huovinen V, Ivaska KK, Kiviranta R, et al. Bone mineral density is increased after a 16-week resistance training intervention in elderly women with decreased muscle strength. Eur J Endocrinol 2016;175:571-82.

39 Yu P-A, Hsu W-H, Hsu W-B, et al. The effects of high impact exercise intervention on bone mineral density, physical fitness, and quality of life in postmenopausal women with osteopenia: a retrospective cohort study. Medicine 2019;98:e14898.

40 Segev-Jacubovski 0, Herman T, Yogev-Seligmann G, et al. The interplay between gait, falls and cognition: can cognitive therapy reduce fall risk? Expert Rev Neurother 2011;11:1057-75.

41 Springer S, Giladi N, Peretz C, et al. Dual-tasking effects on gait variability: the role of aging, falls, and executive function. Mov Disord 2006;21:950-7.

42 Snijders AH, Verstappen CC, Munneke M, et al. Assessing the interplay between cognition and gait in the clinical setting. J Neural Transm 2007;114:1315-21.

43 Hewston P, Kennedy CC, Borhan S, et al. Effects of dance on cognitive function in older adults: a systematic review and meta-analysis. Age Ageing 2021;50:1084-92

44 Granacher U, Muehlbauer T, Bridenbaugh SA, et al. Effects of a salsa dance training on balance and strength performance in older adults. Gerontology 2012;58:305-12.

45 Eyigor S, Karapolat H, Durmaz B, et al. A randomized controlled trial of Turkish folklore dance on the physical performance, balance, depression and quality of life in older women. Arch Gerontol Geriatr 2009:48:84-8.

46 Holmerová I, Machácová K, Vanková H, et al. Effect of the exercise dance for seniors (EXDASE) program on lower-body functioning among institutionalized older adults. J Aging Health 2010;22:106-19.

47 Coubard OA, Duretz $S$, Lefebvre $V$, et al. Practice of contemporary dance improves cognitive flexibility in aging. Front Aging Neurosci 2011;3:13.

48 Kattenstroth J-C, Kalisch T, Holt S, et al. Six months of dance intervention enhances postural, sensorimotor, and cognitive performance in elderly without affecting cardiorespiratory functions. Front Aging Neurosci 2013;5:5.

49 Merom D, Mathieu E, Cerin E, et al. Social dancing and incidence of falls in older adults: a cluster randomised controlled trial. PLoS Med 2016;13:e1002112.

50 Mattle M, Chocano-Bedoya PO, Fischbacher M, et al. Association of Dance-Based Mind-Motor activities with falls and physical function among healthy older adults: a systematic review and meta-analysis. JAMA Netw Open 2020:3:1-19.

51 Fong Yan A, Cobley S, Chan C, et al. The effectiveness of dance interventions on physical health outcomes compared to other forms of physical activity: a systematic review and meta-analysis. Sports Med 2018;48:933-51.

52 Baert V, Gorus E, Mets T, et al. Motivators and barriers for physical activity in older adults with osteoporosis. J Geriatr Phys Ther 2015;38:105-14.

53 Picorelli AMA, Pereira DS, Felício DC, et al. Adherence of older women with strength training and aerobic exercise. Clin Interv Aging 2014;9:323.

54 Seguin RA, Economos CD, Palombo R, et al. Strength training and older women: a cross-sectional study examining factors related to exercise adherence. J Aging Phys Act 2010;18:201-18.

55 Franco MR, Sherrington C, Tiedemann A, et al. Effect of senior dance (DanSE) on fall risk factors in older adults: a randomized controlled trial. Phys Ther 2020;100:600-8.

56 Society AG, Society G, Of AA, et al. Guideline for the prevention of falls in older persons. J Am Geriatr Soc 2001;49:664-72.

57 Chan A-W, Tetzlaff JM, Altman DG, et al. Spirit 2013 statement: defining standard protocol items for clinical trials. Ann Intern Med 2013;158:200-7.

58 Schulz KF, Altman DG, Moher D, et al. Consort 2010 statement: updated guidelines for reporting parallel group randomised trials. BMC Med, . 2010, . ;8:18.

59 Sundhedsstyrelsen. ÆEldres Sundhed OG Trivsel. Sundhedsstyrelsen 2019:6-108

60 Strøbæk Let al. Sygdomsbyrden i Danmark - Ulykker, selvskade og selvmord. 1st ed. Statens Institut for Folkesundhed, Syddansk Universitet for Sundhedsstyrelsen, 2016.

61 Lægemiddelstyrelsen. Bivirkninger OG sikkerhed ved medicinsk cannabis, 2019. Available: https://laegemiddelstyrelsen.dk/da/special/medicinsk-cannabis/borgere/ bivirkninger-og-sikkerhed-ved-medicinsk-cannabis/

62 Cummings SR, Bates D, Black DM. Clinical use of bone densitometry: scientific review. JAMA 2002:288:1889-97.

63 Morse JM, Morse RM, Tylko SJ. Development of a scale to identify the Fall-Prone patient. Can J Aging 1989;8:366-77.

64 Hendrich AL, Bender PS, Nyhuis A. Validation of the Hendrich II fall risk model: a large concurrent case/control study of hospitalized patients. App/ Nurs Res 2003;16:9-21.

65 Estabrooks PA, Carron AV. Group cohesion in older adult exercisers: prediction and intervention effects. J Behav Med 1999;22:575-88 
66 Ageberg E, Link A, Roos EM. Feasibility of neuromuscular training in patients with severe hip or knee oa: the individualized goal-based NEMEX-TJR training program. BMC Musculoskelet Disord 2010;11:126.

67 Lamb SE, Jørstad-Stein EC, Hauer K, et al. Development of a common outcome data set for fall injury prevention trials: the prevention of falls network Europe consensus. J Am Geriatr Soc 2005;53:1618-22.

68 Leere JS, Karmisholt J, Robaczyk M, et al. Denosumab and cinacalcet for primary hyperparathyroidism (DENOCINA): a randomised, double-blind, placebo-controlled, phase 3 trial. Lancet Diabetes Endocrinol 2020;8:407-17.

69 Drewes AM, Helweg-Larsen S, Petersen P, et al. Mcgill pain questionnaire translated into Danish: experimental and clinical findings. Clin J Pain 1993;9:80-7.

70 Washburn RA, Smith KW, Jette AM, et al. The physical activity scale for the elderly (PASE): development and evaluation. J Clin Epidemiol 1993;46:153-62.

71 Washburn RA, McAuley E, Katula J, et al. The physical activity scale for the elderly (PASE): evidence for validity. J Clin Epidemiol 1999;52:643-51.

72 Montero-Odasso M, Almeida QJ, Bherer L, et al. Consensus on shared measures of mobility and cognition: from the Canadian Consortium on neurodegeneration in aging (CCNA). The Journals of Gerontology: Series A 2019;74:897-909.

73 Hirata RP, Arendt-Nielsen L, Graven-Nielsen T. Experimental calf muscle pain attenuates the postural stability during quiet stance and perturbation. Clin Biomech 2010;25:931-7.

74 Rikli RE, Jones CJ. Development and validation of a functional fitness test for Community-Residing older adults. J Aging Phys Act 1999;7:129-61.

75 Franchignoni F, Horak F, Godi M, et al. Using psychometric techniques to improve the balance evaluation systems test: the mini-BESTest. J Rehabil Med 2010;42:323-31.

76 Yingyongyudha A, Saengsirisuwan V, Panichaporn W, et al. The Mini-Balance evaluation systems test (Mini-BESTest) demonstrates higher accuracy in identifying older adult participants with history of falls than do the BESTest, Berg balance scale, or timed up and go test. J Geriatr Phys Ther 2016;39:64-70.

77 Dacey M, Baltzell A, Zaichkowsky L. Older adults' intrinsic and extrinsic motivation toward physical activity. Am J Health Behav 2008;32:570-82.

78 Schutzer KA, Graves BS. Barriers and motivations to exercise in older adults. Prev Med 2004;39:1056-61. 